\title{
EFFECT OF CERTAIN INSECTICIDES AGAINST SUGAR BEET BEETLE Cassida vittata (VILL.) (COLEOPTERA: CHRYSOMELIDAE) INHABITING SUGAR BEET FIELDS.
} Shaheen, F. A. H. ${ }^{*}$; A. A. A. Said ${ }^{\star}$; E. A. H. Sherief ${ }^{\star *}$ and H. A. M. Fouad * Pesticides Dept., Fac. of Agric., Mansoura Univ.

** Plant Protection Res. Inst., Agric. Res. Center., Dokki-Giza, Egypt.

\begin{abstract}
The objective of present work was to compare the field efficiency of three tested pesticides, Selecron (profenofos), Marshal (carbosulfan) and Radiant (spinetoram), against larvae and adults of the tortoise beetle Cassida vittata (Vill.) inhabiting sugar beet fields in Sharkia Governorate during 2008/2009 and 2009/2010 sugar beet growing seasons. Data obtained revealed that when mortality rates were considered, Selecrone and Marshal were the most efficient compounds against larvae and adults of $C$. vittata. However, Radiant demonstrated a moderate toxic effect.

Marshal and Selecrone showed an increase in sugar percentage $\quad(16.75 \%$ and $14.5 \%$ respectively) and total soluble solids (TSS \%) (18\%, $17 \%$ respectively) in the first season, while in the second season the increase in sugar percentage were ( $19.8 \%$ and $19.10 \%$ respectively), and TSS \% was (22\% and $20 \%$, respectively). In the second season Radiant showed less percentages of sugar and (TSS) which are $16.70 \%$ and $18.50 \%$, respectively.

Keywords: Beta vulgaris, Cassida vittata, insecticides, profenofos, carbosulfan, spinetoram, sugar percentage, total soluble solids.
\end{abstract}

\section{INTRODUCTION}

Sugar beet, Beta vulgaris L. is one of two strategic sugar crops in Egypt. Because of its lower consumption of irrigation water and its shorter growing season, sugar beet is planted in extending area with decreasing area of sugar cane crop in Egypt. Sugar beet plants attract a considerable number of insect pests among most important of them is the tortoise beetle, Cassida vittata (Coleoptera: Chrysomelidae).

Mahmoud et al. (1973) and Youssef (1994) recorded that larvae and adults of $C$. vittata are leaf feeders. Crop loss occurs due to leaf feeding and reduction in sugar content of infested plants. Abo-Aiana (1991) mentioned that $C$. vittata reduced both quantity and quality of sugar beet. El-Sebae et al. (1985) found that lannate alone had no reliable effect against C. vittata control, but mixtures of lannate/dimilin or lannate/sir-8514 were the most effective. Hano (1983); Ali et al. (1993) and Saleh (1994) stated that, some conventional insecticides, i.e. pirimiphos-methyl, monocrotophos, profenofos, methomyl were comparatively more effective against $C$. vittata under field conditions. El-Khouly and Omar (2002) evaluated the efficiency of profenofos, carbosulfan and chlorfenapyr against eggs, larvae, pupae and adults of the tortoise beetle Cassida vittata (Vill.). 
The present work was carried out to evaluate certain conventional and untraditional insecticides against $C$. vittata infesting sugar beet plants and their effect on Juice quality, root yield and sugar yield of sugar beet at Sharkia Governorate during two successive growing seasons.

\section{MATERIALS AND METHODS}

\section{1- Field experiment:}

Field studies were conducted at Kafr El-hamam village, Zagazig district Sharkia Governorate, during the two successive seasons, 2008/2009 and 2009/2010. The sugar beet variety used was Baraca, sowed at Mid. of November. The normal agriculture practice was followed.

\section{2- Pesticides treatments:}

Three treatments including control were used in the first season. While, in the second season four treatments including control in complete randomized block design were used. Each treatment has been divided into four plots (replicates). The area of each plot was $42 \mathrm{~m}^{2}\left(6 \times 7 \mathrm{~m}^{2}\right)$; so that the area of each treatment was $168 \mathrm{~m}^{2}\left(6 \times 28 \mathrm{~m}^{2}\right)$.

Each chemical was used separately in a single treatment contain four replicates. Sprayer provided with motor spray (20 liter) (Kubota) has proved to be sufficient to give good coverage of the tested sugar beet plants. Spraying was carried out when the number of insect pests were increased in the end of sugar beet season to save sugar beet plants from severe infestations. Sugar beet was sprayed with the tested insecticides after five months (April) from sowing. Normal agricultural practices were followed; barriers were left between treatments to avoided drift.

Five sugar beet plants were chosen randomly from each replicate

plants per treatment) to estimate the number of $C$. vittata adult and larvae before and after 1, 3, 5, 7, 9 and 11 days of spraying.

Percentage of reduction (R \%) was estimated according to the formula of Henderson and Tilton (1955) as follows:

$$
\% R=1-\left(\frac{\text { Insect No in check before spray }}{\text { Insect No in check after spray }} X \frac{\text { Insect No in treatment after spray }}{\text { Insect No in treatment before spray }}\right) X 100
$$

3- Tested compounds:

a. Marshal (25\% WP) $(800 \mathrm{gm} / \mathrm{fed})$ :

Common name: Carbosulfan.

Chemical name: 2,3-dihydro-2,2-dimethyl-7-benzofuranyl[(dibutylamino) thio]

Empirical formula: $\mathrm{C} 20 \mathrm{H} 32 \mathrm{~N} 2 \mathrm{O} 3 \mathrm{~S}$ methylcarbamate.

b. Selecron $\left(72 \%\right.$ EC) $\left(750 \mathrm{~cm}^{3} / \mathrm{fed}\right)$ :

Common name: profenofos.

Chemical name: $O$-(4-bromo-2-chlorophenyl) O-ethyl S-propyl phosphorothioate.

Empirical formula: $\mathrm{C} 11 \mathrm{H} 15 \mathrm{Br} \mathrm{Cl} \mathrm{O} 3 \mathrm{PS}$.

c. Radiant (12\% SC) $\left(100 \mathrm{~cm}^{3} /\right.$ fed):

Common name: spinetoram. 
Chemical name: $(2 R, 3 \mathrm{a} R, 5 \mathrm{a} R, 5 \mathrm{~b} S, 9 S, 13 S, 14 R, 16 \mathrm{a} S, 16 \mathrm{~b} R)-2-[(6-$ deoxy-3 Oethyl-2,4-di-O-methyl-a-L mannopyranosyl)oxy]-13[[(2R,5S,6R)-5-(dimethylamino)tetrahydro-6-methyl$2 H$-pyran-2-yl]oxy]-9-ethyl 2,3,3a, $4,5,5 a, 5 b, 6,9,10,11,12,13,14,16 a, 16 b-$ hexadecahydro-14-methyl-1 $\mathrm{H}$-as-indaceno[3,2d]oxacyclododecin-7,15-dione

Mixed with: $\quad(2 S, 3 \mathrm{a} R, 5 \mathrm{a} S, 5 \mathrm{~b} S, 9 S, 13 S, 14 R, 16 \mathrm{a} S, 16 \mathrm{~b} S)-2-[(6-$ deoxy-3-Oethyl-2,4-di-O-methyl-a-L-mannopyranosyl)oxy]-13[[(2R,5S,6R)-5-(dimethylamino)tetrahydro-6-methyl$2 H$-pyran-2-yl]oxy]-9-ethyl2,3,3a,5a,5b,6,9,10,11,12,13,14,16a,16btetradecahydro-4,14-dimethyl-1H-as-indaceno[3,2d] oxacyclododecin-7,15-dione.

Empirical formula: $\mathrm{C}_{42} \mathrm{H}_{69} \mathrm{NO}_{10}+\mathrm{C}_{43} \mathrm{H}_{69} \mathrm{NO}_{10}$.

4- Juice quality, root yield and sugar yield determinations:

For estimating the effect of tested insecticides on sugar beet yield quantity and percentage of sugar at harvest, 20 plants were taken (from each treatment) and the leaves were cut-off. The roots were cleaned and weighted to calculate the root yield. Sugar extractable was determined at the Department of Pesticide, Faculty of Agriculture, Mansoura University. Juice quality and sugar yield were calculated as follow:

1. Total soluble solids (TSS \%) which was determined using handle refractometer. according to Simon et al. (1980).

2. Sucrose percentage (\%) was determined in fresh roots polarmetrically using lead acetate according to the methods of Le-Docte (1927).

3. Purity percentage was calculated according to the following formula: apparent purity $\%=$ sucrose $\% /$ TSS $\% \times 100$. According to Poschenok (1976).

4. Sugar yield $($ ton $/ \mathrm{fed})=$ root yield $X$ sugar extractable $\%$.

5- Statistical analysis:

Statistical analysis were carried out to determine the differences between treatment and days after spraying by using one way analysis of variance (ANOVA) (Costat, 1990). Duncan's multiple range test (Duncan, 1955) was applied at $5 \%$ probability level.

\section{RESULTS AND DISCUSSION}

\section{1- Efficiency of tested pesticides against adults and larvae of Cassida vittata:}

\section{A. season $2008 / 2009$ :}

Results presented in table (1) showed that Marshal was more effective than Selecron against adults of C. vittata recording $75.15 \%$ and $99.06 \%$ reduction while, Selecron caused $67.85 \%$ and $95.05 \%$ as initial kill (after one day) and mean residual effect, respectively.

Regarding the reduction percent at different time intervals, Marshal and Selecron caused (99.23\% and 97.36\%), (100\% and 97.24\%), (100\% and 
$95.68 \%)$ and (100\% and 93.96\%) reduction after 5, 7, 9 and 11 days of application, respectively.

Data in table (2) demonstrated that tested materials had generally great effect against larvae of $C$. vittata, where Marshal showed higher initial effect than Selecron $(93.05 \%$ and $81.48 \%$ reduction, respectively).

There were no significant differences between Marshal and Selecron as residual effect caused (95.19\% and $100 \%),(96.04 \%$ and $94.72 \%)$ and $(96.79 \%$ and $95.65 \%)$ reduction at 7,9 and 11 days after application, respectively. According to the mean of residual effect tested insecticides caused $98.07 \%$ and $97.60 \%$ for Selecrone and Marshal, respectively.

\section{B. season 2009/2010:}

As indicated in the result given in table (3) Radiant and Selecron were more effective than Marshal against adults of $C$. vittata in which reduction percentages were $64.80 \%, 64.25 \%$ and $41.33 \%$ after one day of spraying (initial kill), respectively.

With the respect to residual effect, Selecron had the highest effect, recording $100 \%$ reduction after $5,7,9,11$ days of pesticides application.

As means of residual effect, tested insecticides were arranged in descending order as follow: Selecron (98.67\%) > Marshal (93.46\%) > Radiant $(90.76 \%)$.

Data in table (4) showed that Selecron was the most effective compound recording $97.66 \%$ and $100 \%$ reduction followed by Marshal $77.38 \%$ and $100 \%$ reduction as initial kill (after one day) and residual effect, respectively. While, Radiant was the least potent one where caused $75.25 \%$ reduction as initial kill (after one day) and $99.51 \%$ reduction as residual effect. Comparing, the effect of the tested materials on larvae and adults of C. vittata, data in fig (1) showed that the tested insecticides were more effective against larvae than the adult of $C$. vittata during the two seasons; it was more clearly in season 2009/2010 than 2008/2009.

These results are in agreement with data obtained by Bassyony and Bleih (1996) who found that Marshal and Selecron were the best compounds in reducing the adults of $C$. vittata. El-khouly (1998) stated that Selecrone was the most effective insecticides against the immature stages of $C$. vittata. $\mathrm{He}$ added that successful reduction in adult population also recorded by spraying infested sugar beet plants with Selecron and Marshal. Abo El-Naga (2004) found that Selecrone was the most effective insecticides followed by Marshal as they induced high initial and long residual effect against both adults and larvae of C. vittata. Gehan (2009) found that Marshal was the most effective against $C$. vittata compared with the other four compounds where, Marshal caused $82.3 \%$ decrease in the adult population followed by Achook (68.5\%). the efficiency of tested compounds can be arranged as follows: Marshal > Achook > Bancol > Pymetozine > Alkanz. 
J. Plant Prot. and Pathology, Mansoura Univ., Vol. 2 (6), June, 2011

$1-2$

601 
Shaheen, F. A. H. et al.

3-4

602 

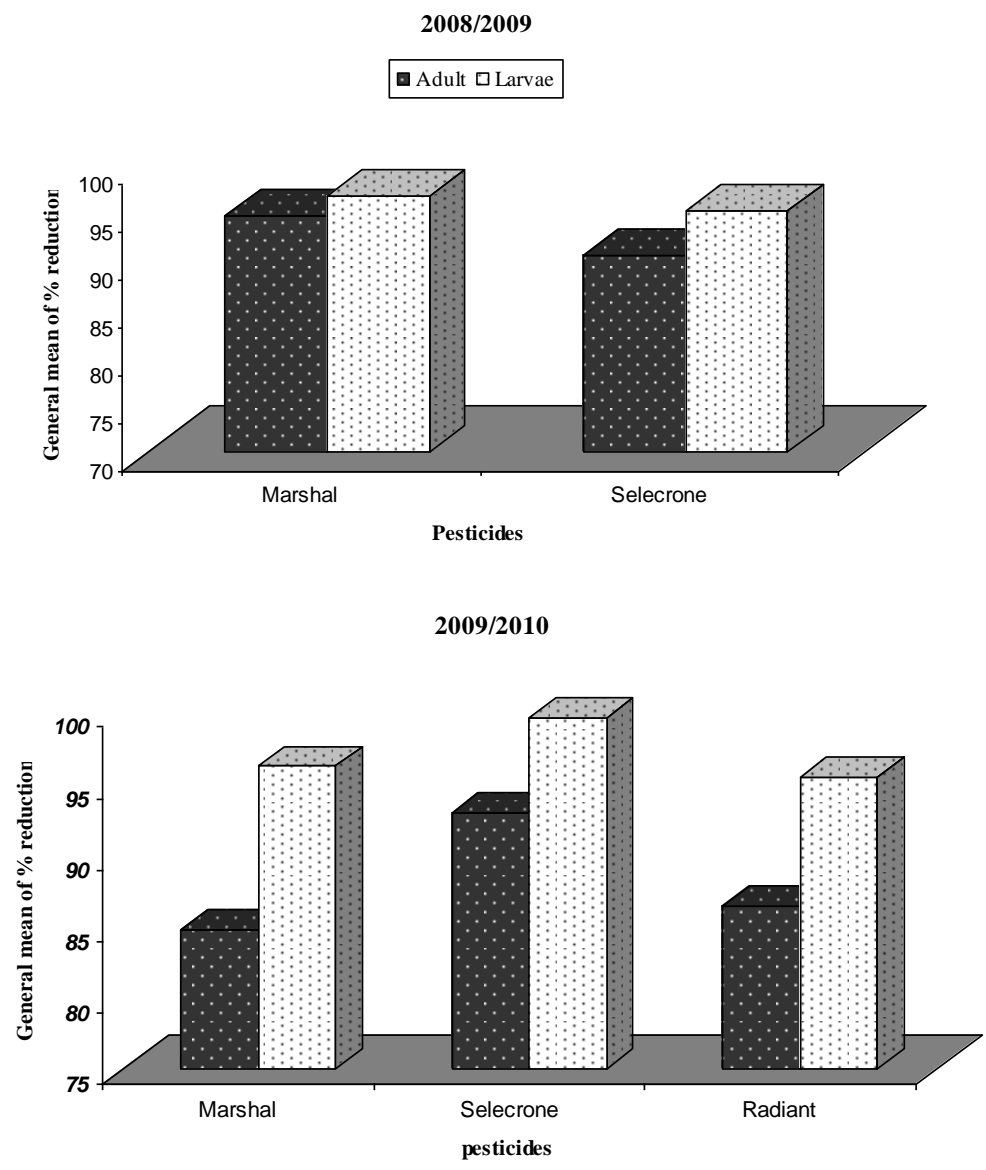

Fig. (1): General mean of reduction in sugar beet beetle adults and larvae of Cassida vittata on sugar beet leaves during 2008/2009 and 2009/2010 seasons.

\section{2- Efficiency of insecticides on Juice quality, Root yield and Sugar} yield:

a. Season 2008/2009:-

Data in table (5) indicated that there was significant increase in percent of sugar extractable, root yield, and sugar yield in sugar beet plants treated with Marshal compared with Control. On the other hand there were no significant differences in case of Selecron treatment. As shown in fig (1) Marshal and Selecron showed an increase in sugar percentage $(16.75 \%$ and $14.5 \%$ respectively) and total soluble solids (TSS) (18\%, $17 \%$ respectively) compared with Control which showed (12.65\% and $14.5 \%)$ for percentages of sugar and (TSS), respectively. The highest juice purity percentage obtained with Marshal was $93.05 \%$ compared with Control which 
Shaheen, F. A. H. et al.

was $87.24 \%$, while Selecron was significantly the lowest one in purity percentage.

Data also stated that Marshal increased the sugar and roots yields (Ton / fed.) comparing with other treatments.

Table (5): The side effect of tested insecticides on Juice quality, Root yield and Sugar yield of treated sugar beet plants during season 2008/2009.

\begin{tabular}{|l|c|c|c|c|c|c|}
\hline insecticides & $\begin{array}{c}\text { Root weight } \\
(\mathbf{k g}) / 20 \text { plants }\end{array}$ & $\begin{array}{c}\text { Sucrose } \\
(\%)\end{array}$ & $\begin{array}{c}\text { TSS } \\
(\%)\end{array}$ & $\begin{array}{c}\text { Purity } \\
(\%)\end{array}$ & $\begin{array}{c}\text { Roots } \\
\text { yield } \\
\text { Ton/Fed }\end{array}$ & $\begin{array}{c}\text { Sugar } \\
\text { yield } \\
\text { Ton/Fed }\end{array}$ \\
\hline Selecron & 22.50 & 14.50 & 17.0 & 85.29 & 31.500 & 4.88 \\
\hline Marshal & 30.65 & 16.75 & 18.0 & 93.05 & 42.910 & 7.61 \\
\hline Control & 20.90 & 12.65 & 14.5 & 87.24 & 29.260 & 3.70 \\
\hline L.S.D. & & & & & \\
\hline
\end{tabular}

TSS $=$ total soluble solids.

Table (6): The side effect of tested insecticides on Juice quality, Root yield and Sugar yield of treated sugar beet plants during season 2009/2010.

\begin{tabular}{|l|c|c|c|c|c|c|}
\hline insecticides & $\begin{array}{c}\text { Root weight } \\
\text { (kg)/20plants }\end{array}$ & $\begin{array}{c}\text { Sucrose } \\
\text { (\%) }\end{array}$ & $\begin{array}{c}\text { TSS } \\
(\%)\end{array}$ & $\begin{array}{c}\text { Purity } \\
(\%)\end{array}$ & $\begin{array}{c}\text { Roots yield } \\
\text { Ton/Fed }\end{array}$ & $\begin{array}{c}\text { Sugar } \\
\text { yield } \\
\text { Ton/Fed }\end{array}$ \\
\hline Selecron & 26.35 & 19.10 & 20.00 & 95.50 & 36.890 & 7.05 \\
\hline Marshal & 32.83 & 19.80 & 22.00 & 90.00 & 45.962 & 9.10 \\
\hline Radiant & 23.60 & 16.70 & 18.50 & 90.27 & 33.040 & 5.52 \\
\hline Control & 19.15 & 14.70 & 16.75 & 87.76 & 26.810 & 3.94 \\
\hline L.S.D. & & & & & \\
\hline
\end{tabular}

TSS $=$ total soluble solids.

\section{b. Season 2009/2010:-}

Data in table (6) and fig (2) illustrated that Marshal and Selecron increased the percentage of sugar extractable significantly than Control (19.8\%, 19.10\% and $14.70 \%$, respectively). According to the sugar and (TSS) percentages the tested insecticides were arranged in descending order as the follows: Marshal > Selecron > Radiant. On the other hand, the juice purity percentage was arranged in descending order as the follows: Selecron > Radiant $>$ Marshal.

Data also stated that Marshal and Selecron increased the sugar and roots yields (Ton / fed.) comparing with Control which recorded 9.10, 7.05 and 3.94 (ton/ fed.), respectively. 

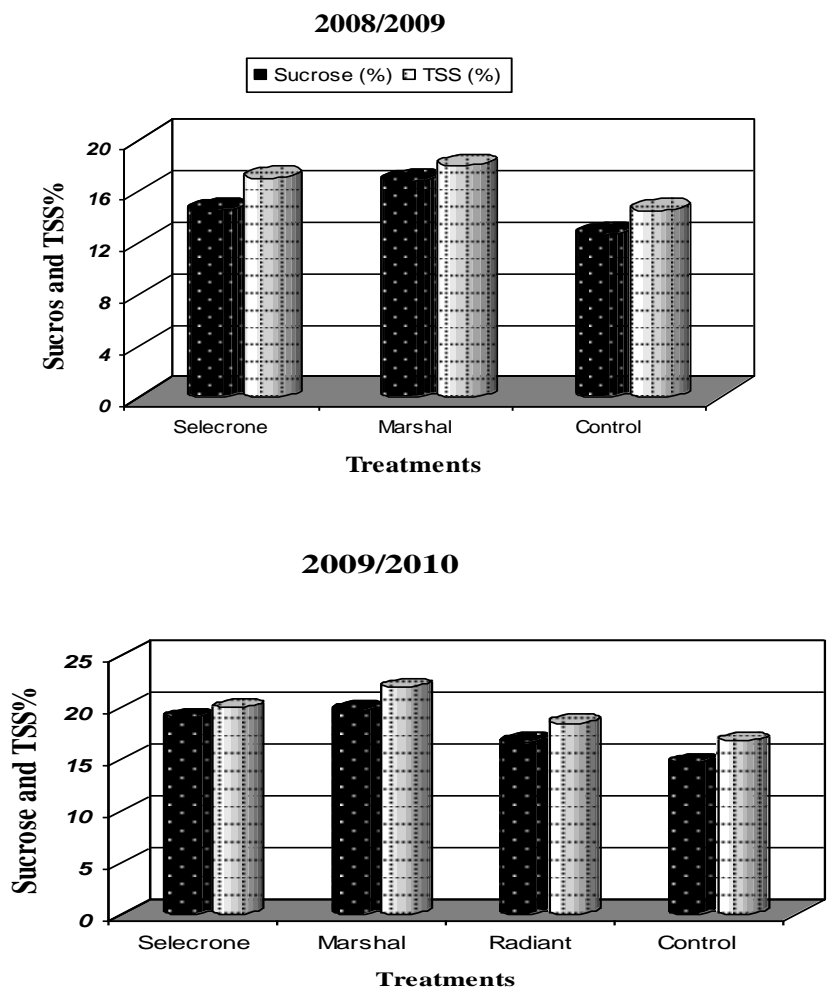

Fig. (2): Effect of certain insecticides on sugar content and total soluble solids during 2008/2009 and 2009/2010.

\section{REFERENCES}

Abo-Aiana, R. A. D. (1991). Studies on pests of sugar beet in Kafr El-Sheikh. Ph. D. Thesis, Fac. of Agric, Tanta Univ., 171 pp.

Abo El-Naga, A. M. M. (2004). Ecological studies and integrated control of the sugar beet beetle, Cassida vittata Vill. M. Sc. Thesis, Fac. Agric; Tanta Univ., $129 \mathrm{pp}$.

Ali, F.A.; M.M.A. Mahgoub; S.I. El-Deib and M.A. El-Hamady (1993). Ecological studies on the tortoise beetle, Cassida vittata Vill., and the efficacy of certain chemicals in controlling this insect in sugar-beet plants. J. Agric. Sci., Mansoura Univ., Egypt, 18 (6): 1813-1824.

Bassyouny, A. M. and S. B. Bleih (1996). Sowing dates, seasonal fluctuations and chemical control against the main insects attacking sugar beet. Alex. Sci. Exch., Egypt, 17 (3): 283 - 296.

Costat Statistical Software (1990). Microcomputer program analysis version 4.20, Cohort Software, Berkeley, CA. 
Duncan, D.B. (1955). Multiple range and multiple F-tests. Biometrics, 11: 142.

El-Khouly, M. I. I. (1998). Ecological studies and control of the tortoise beetle, Cassida vittata Villers in sugar beet ecosystem. Ph. D. Thesis, Fac. Agric, Al-Azhar Univ., Egypt, 183 pp.

El-Khouly M.I. and B.A. Omar (2002). The efficiency of some insecticides on tortoise beetle, Cassida Vittata Vill., inhabiting sugar beet fields. Egypt. J. Agric. Res., 80 (2): 697-708.

El-Sebae, A.H; S.E. Negm; A.A.A. Said; A.A.Abd El-Ghany and M.A.M.Samy. (1985). Efficiency of field sprayed pesticides and their mixtures against some sugar beet pests. J. agric. Sci. Mansoura Univ. 12(4): 1333-1340.

Gehan A.Y. (2009). Use of some relatively safe compounds for controlling of the tortoise beetle, Cassida vittata (Vill.) in sugar beet crop. Research Journal of Agriculture and Biological Sciences, (2009). 5(1): 24-28.

Hano, G. (1983). Damage by Cassida nebulosa in sugar-beet possibilities of control, (C.F. R.A.E., 19(5): 234, pp. 747).

Henderson, C.F. and E.W. Tilton. (1955). Test with acaricides against the brown wheat mite. J. Econ. Entomol., 84: 157-161.

Le-Docte, A. (1927). Commercial determination of sugar beet root using the Sachr Le-Docte processes. Int. Sugar J., 29: 488 - 492. (C.F. Sugar beet nutrition, Applied Sciences Publishers LTD, London, A.P. Draycott).

Mahmoud A.; M.A. Assem; K.H. Youssef; A.A. Meshour; G.A.I. El-Imam (1973). Studies on the main pests by using some recent insecticides. In abstracts of the $4^{\text {th }}$ vegetable research conference, Alexandria University, Alexandria, 2-4 September 1973, p 60.

Poschenok (1976). Biochemical analysis of fruit and vegetable products. TataMc graw-Hill publishing company limited, New Delhi pp, 634 650.

Saleh, A.Y. (1994). Insecticide testes to control the tortoise beetle, Cassida vittata (Vill.) in sugar beet crop. Assiut. J. Agric. Sci. 25(4): 197-202.

Simon, P.W.; C.E. Peterson and R.C. Lindsay (1980). Correlations between sensory and objective parameters of college flavor, J. Agric. Food chem. 28: $559-562$.

Youssef A.E. (1994). Studies on certain insects attacking sugar beet. Ph.D. Thesis, Faculty of Agriculture, Tanta University, Kafr El-Sheikh. 
تأثير بعض المبيدات الحشرية ضد حشرة خنفسـاء البنجر السـلحفائية التى تقطن حقول بنجر السكر المبري

فؤاد عبدالله حسام الدين شاهين *, على على عبدالهادى سعيد *, السيد على حسن شريف

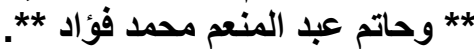

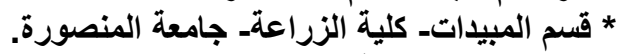

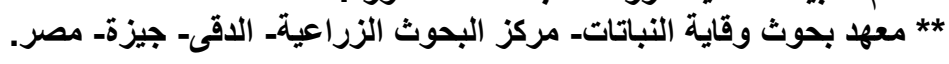

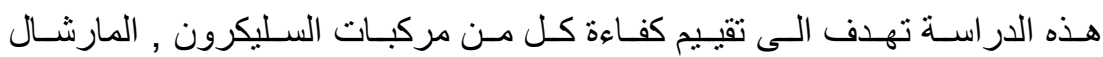

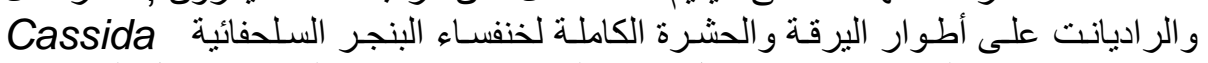
vittata (Vill.) 2008

أظهرت النتائج أن السليكرون و المارشـال كانوا أكثر المركبات كفاءة على اليرقة الئة

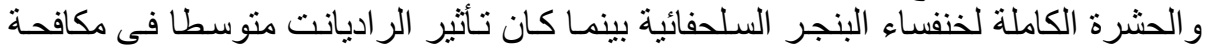

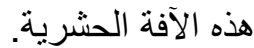

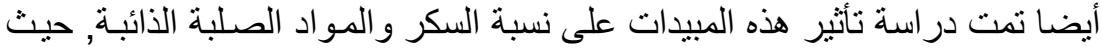

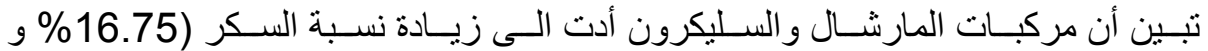

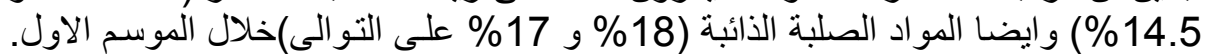

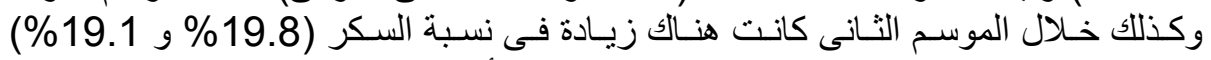

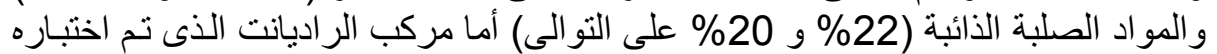

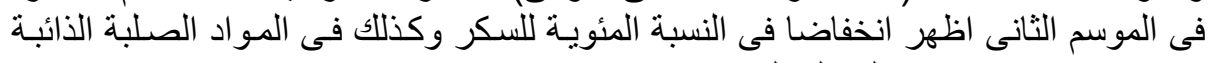
(16.7\% و 18.5 \% على التوالى).

كلية الزراعة - جامعة المنصورة مركز البحوث الزراعية

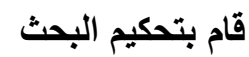

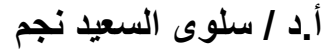

أ.د ا وحيد محمود حسين دسوقى نجي الدي 
Shaheen, F. A. H. et al.

608 
J. Plant Prot. and Pathology, Mansoura Univ., Vol. 2 (6): 597 - 607, 2011

Table (1): Efficiency of tested pesticides against adult of Cassida vittata on sugar beet leaves during 2008/2009 at Sharkia Governorate.

\begin{tabular}{|c|c|c|c|c|c|c|c|c|c|c|c|c|c|c|c|c|}
\hline \multirow{4}{*}{ Treatment } & \multicolumn{13}{|c|}{ \%Reduction at time intervals } & \multirow{4}{*}{$\begin{array}{c}\text { Mean of } \\
\text { residual } \\
\text { effect }\end{array}$} & \multirow{4}{*}{$\begin{array}{c}\text { General } \\
\text { mean of } \\
\% \\
\text { reduction }\end{array}$} & \multirow{4}{*}{$\begin{array}{l}\text { L.S.D } \\
0.05\end{array}$} \\
\hline & \multirow{2}{*}{\multicolumn{3}{|c|}{\begin{tabular}{c|c|}
$\begin{array}{c}\text { One day } \\
\text { before }\end{array}$ & $\begin{array}{c}\text { Initial effect } \\
\text { (after one day) }\end{array}$ \\
application
\end{tabular}}} & \multicolumn{10}{|c|}{ In days (residual effect) } & & & \\
\hline & & & & \multicolumn{2}{|c|}{3} & \multicolumn{2}{|c|}{5} & \multicolumn{2}{|c|}{\begin{tabular}{|l|l}
7 \\
\end{tabular}} & \multicolumn{2}{|c|}{9} & \multicolumn{2}{|c|}{11} & & & \\
\hline & Mean & Mean & $\begin{array}{c}\text { Reduc } \\
\text { tion }\end{array}$ & Mean & $\begin{array}{c}\text { Reduc } \\
\text { tion }\end{array}$ & Mean & $\begin{array}{c}\text { Reduc } \\
\text { tion }\end{array}$ & Mean & \begin{tabular}{|c|} 
Reduc \\
tion
\end{tabular} & Mean & $\begin{array}{c}\text { Reduc } \\
\text { tion }\end{array}$ & Mean & $\begin{array}{c}\text { Reduc } \\
\text { tion }\end{array}$ & & & \\
\hline Marshal & $\begin{array}{c}34.50 \mathrm{~A}^{\mathrm{a}} \\
\pm 8.10\end{array}$ & $\begin{array}{l}8.00 \mathrm{~B}^{\mathrm{b}} \\
\pm 6.16\end{array}$ & 75.15 & $\begin{array}{l}1.25 \mathrm{~B}^{\mathrm{C}} \\
\pm 1.25\end{array}$ & 96.08 & $\begin{array}{c}0.25 \mathrm{~B}^{\mathrm{c}} \\
\pm 0.5\end{array}$ & 99.23 & $\begin{array}{l}0.00 \mathrm{~B}^{\mathrm{c}} \\
\pm 0.00\end{array}$ & 100 & $\begin{array}{l}0.00 \mathrm{~B}^{\mathrm{c}} \\
\pm 0.00\end{array}$ & 100 & $\begin{array}{l}0.00 \mathrm{~B}^{\mathrm{c}} \\
\pm 0.00\end{array}$ & 100 & 99.06 & 95.08 & $5.71^{* *}$ \\
\hline Selecrone & \begin{tabular}{|c|}
$30.00 \mathrm{~A}^{\mathrm{a}}$ \\
\pm 2.16 \\
\end{tabular} & $\begin{array}{c}9.00 \mathrm{~B}^{\mathrm{b}} \\
\pm 3.55 \\
\end{array}$ & 67.85 & $\begin{array}{l}2.50 \mathrm{~B}^{\mathrm{C}} \\
\pm 3.10 \\
\end{array}$ & 90.99 & $\begin{array}{l}0.75 \mathrm{~B}^{\mathrm{C}} \\
\pm 0.95 \\
\end{array}$ & 97.36 & $\begin{array}{l}0.75 \mathrm{~B}^{\mathrm{c}} \\
\pm 0.95 \\
\end{array}$ & 97.24 & $\begin{array}{l}1.25 \mathrm{~B}^{\mathrm{c}} \\
\pm 1.25 \\
\end{array}$ & 95.68 & $\begin{array}{l}1.75 \mathrm{~B}^{\mathrm{c}} \\
\pm 1.70 \\
\end{array}$ & 93.96 & 95.05 & 90.51 & $3.21^{* *}$ \\
\hline Control & $\begin{array}{c}30.00 \mathrm{~A}^{\mathrm{a}} \\
\pm 9.05 \\
\end{array}$ & $\begin{array}{c}28.00 \mathrm{~A}^{\mathrm{a}} \\
\pm 8.86 \\
\end{array}$ & - & $\begin{array}{c}27.75 \mathrm{~A}^{\mathrm{a}} \\
\pm 9.06 \\
\end{array}$ & - & $\begin{array}{c}28.50 \mathrm{~A}^{\mathrm{a}} \\
\pm 7.14 \\
\end{array}$ & - & $\begin{array}{c}27.25 \mathrm{~A}^{\mathrm{a}} \\
\pm 7.04 \\
\end{array}$ & - & $\begin{array}{c}29.00 \mathrm{~A}^{\mathrm{a}} \\
\pm 6.87 \\
\end{array}$ & - & $\begin{array}{c}29.00 \mathrm{~A}^{\mathrm{a}} \\
\pm 7.11 \\
\end{array}$ & - & - & - & $11.67 \mathrm{NS}$ \\
\hline $\begin{array}{l}\text { L.S.D } \\
0.05\end{array}$ & $11.39 N . S$ & $10.80^{\star *}$ & - & $8.93^{\star *}$ & - & $6.67^{\star *}$ & - & $6.56^{\star \star}$ & - & $6.45^{\star \star}$ & - & $6.76^{\star \star}$ & - & - & - & - \\
\hline
\end{tabular}

*Means followed the same capital letter in a column for different pesticides or s

significantly different at $5 \%$ level of Probability (Duncan's Multiple Rang Test).

Table (2): Efficiency of tested pesticides against larvae of Cassida vittata on sugar beet leaves during 2008/2009 at Sharkia Governorate.

\begin{tabular}{|c|c|c|c|c|c|c|c|c|c|c|c|c|c|c|c|c|}
\hline \multirow{4}{*}{ Treatment } & \multicolumn{13}{|c|}{ \%Reduction at time intervals } & \multirow{4}{*}{$\begin{array}{c}\text { Mean of } \\
\text { residual } \\
\text { effect }\end{array}$} & \multirow{4}{*}{$\begin{array}{c}\text { General } \\
\text { mean of } \\
\% \\
\text { reduction }\end{array}$} & \multirow{4}{*}{$\begin{array}{l}\text { L.S.D } \\
0.05\end{array}$} \\
\hline & \multirow{3}{*}{$\begin{array}{c}\begin{array}{c}\text { One day } \\
\text { before } \\
\text { application }\end{array} \\
\text { Mean }\end{array}$} & \multirow{2}{*}{\multicolumn{2}{|c|}{$\begin{array}{c}\text { Initial effect } \\
\text { (after one day) }\end{array}$}} & \multicolumn{10}{|c|}{ In days (residual effect) } & & & \\
\hline & & & & \multicolumn{2}{|c|}{3} & \multicolumn{2}{|c|}{5} & \multicolumn{2}{|c|}{7} & \multicolumn{2}{|c|}{9} & \multicolumn{2}{|c|}{11} & & & \\
\hline & & Mean & $\begin{array}{c}\text { Reduct } \\
\text { ion }\end{array}$ & Mean & $\begin{array}{c}\text { Reduc- } \\
\text { tion }\end{array}$ & Mean & $\begin{array}{c}\text { Reduc- } \\
\text { tion }\end{array}$ & Mean & $\begin{array}{c}\text { Reduc- } \\
\text { tion }\end{array}$ & Mean & $\begin{array}{c}\text { Reduct } \\
\text { ion }\end{array}$ & Mean & $\begin{array}{c}\text { Reduc- } \\
\text { tion }\end{array}$ & & & \\
\hline Marshal & $\begin{array}{l}4.00 \mathrm{~B}^{\mathrm{a}} \\
\pm 2.16\end{array}$ & $\begin{array}{c}0.25 \mathrm{~B}^{\mathrm{b}} \\
\pm 0.5\end{array}$ & 93.05 & $\begin{array}{l}0.00 \mathrm{~B}^{\mathrm{b}} \\
\pm 0.00\end{array}$ & 100 & $\begin{array}{c}0.00 \mathrm{~B}^{\mathrm{b}} \\
\pm 0.00\end{array}$ & 100 & $\begin{array}{c}0.25 \mathrm{~B}^{\mathrm{b}} \\
\pm 0.5\end{array}$ & 95.19 & $\begin{array}{c}0.25 \mathrm{~B}^{\mathrm{b}} \\
\pm 0.5\end{array}$ & 96.04 & $\begin{array}{c}0.25 \mathrm{~B}^{\mathrm{b}} \\
\pm 0.5\end{array}$ & 96.79 & 97.60 & 96.85 & $1.32^{\star *}$ \\
\hline Selecrone & $\begin{array}{l}6.00 \mathrm{~B}^{\mathrm{a}} \\
\pm 1.82\end{array}$ & $\begin{array}{l}1.00 \mathrm{~B}^{\mathrm{b}} \\
\pm 0.81\end{array}$ & 81.48 & $\begin{array}{l}0.00 \mathrm{~B}^{\mathrm{b}} \\
\pm 0.00\end{array}$ & 100 & $\begin{array}{l}0.00 \mathrm{~B}^{\mathrm{b}} \\
\pm 0.00\end{array}$ & 100 & $\begin{array}{l}0.00 \mathrm{~B}^{\mathrm{b}} \\
\pm 0.00\end{array}$ & 100 & $\begin{array}{l}0.50 \mathrm{~B}^{\mathrm{b}} \\
\pm 1.00\end{array}$ & 94.72 & $\begin{array}{l}0.50 \mathrm{~B}^{\mathrm{b}} \\
\pm 1.00\end{array}$ & 95.65 & 98.07 & 95.31 & $1.36^{* \star}$ \\
\hline Control & $\begin{array}{c}12.50 \mathrm{~A}^{\mathrm{cd}} \\
\pm 6.45\end{array}$ & $\begin{array}{c}11.25 \mathrm{~A}^{\mathrm{cc}} \\
\pm 6.29\end{array}$ & - & $\begin{array}{l}8.50 \mathrm{~A}^{\mathrm{a}} \\
\pm 4.43 \\
\end{array}$ & - & $\begin{array}{c}13.75 \mathrm{~A}^{\mathrm{bcc}} \\
\pm 1.70\end{array}$ & - & $\begin{array}{c}16.25 \mathrm{~A}^{\mathrm{b}} \\
\pm 1.25\end{array}$ & - & $\begin{array}{r}19.75 \mathrm{~A}^{\mathrm{a}} \\
\pm 4.34\end{array}$ & - & $\begin{array}{r}24.00 \mathrm{~A} \\
\pm 2.94\end{array}$ & - & - & - & $6.41^{* *}$ \\
\hline \begin{tabular}{|l|} 
L.S.D \\
0.05
\end{tabular} & $6.51^{*}$ & $5.87^{* *}$ & - & $4.09^{* *}$ & - & $1.57^{* *}$ & - & $1.25^{\star *}$ & - & $4.14^{* *}$ & - & $2.91^{* *}$ & - & - & - & - \\
\hline
\end{tabular}

$\begin{array}{llllll}0.05 & \\ *\end{array}$ significantly different at $5 \%$ level of Probability (Duncan's Multiple Rang Test). 
Table (3): Efficiency of tested pesticides against adult of Cassida vittata on sugar beet leaves during 2009/2010 at Sharkia Governorate.

\begin{tabular}{|c|c|c|c|c|c|c|c|c|c|c|c|c|c|c|c|c|}
\hline \multirow{4}{*}{ Treatment } & \multicolumn{13}{|c|}{ \%Reduction at time intervals } & \multirow{4}{*}{$\begin{array}{c}\text { Mean of } \\
\text { residual } \\
\text { effect }\end{array}$} & \multirow{4}{*}{$\begin{array}{c}\text { General } \\
\text { mean of } \\
\% \\
\text { reduction }\end{array}$} & \multirow{4}{*}{$\begin{array}{l}\text { L.S.D } \\
0.05\end{array}$} \\
\hline & \multirow{3}{*}{\begin{tabular}{|c|}
$\begin{array}{c}\text { One day } \\
\text { before } \\
\text { application }\end{array}$ \\
Mean \\
\end{tabular}} & \multirow{2}{*}{\multicolumn{2}{|c|}{$\begin{array}{c}\text { Initial effect } \\
\text { (after one day) }\end{array}$}} & \multicolumn{10}{|c|}{ In days (residual effect) } & & & \\
\hline & & & & \multicolumn{2}{|r|}{3} & \multicolumn{2}{|c|}{5} & \multicolumn{2}{|c|}{7} & \multicolumn{2}{|c|}{9} & \multicolumn{2}{|c|}{11} & & & \\
\hline & & Mean & $\begin{array}{c}\text { Reduc- } \\
\text { tion }\end{array}$ & Mean & \begin{tabular}{|c|}
$\begin{array}{c}\text { Reduc- } \\
\text { tion }\end{array}$ \\
\end{tabular} & Mean & \begin{tabular}{|c|}
$\begin{array}{c}\text { Reduc- } \\
\text { tion }\end{array}$ \\
\end{tabular} & Mean & $\begin{array}{c}\text { Reduc- } \\
\text { tion }\end{array}$ & Mean & $\begin{array}{c}\text { Reduc- } \\
\text { tion }\end{array}$ & Mean & $\begin{array}{c}\text { Reduc- } \\
\text { tion }\end{array}$ & & & \\
\hline Marshal & $\begin{array}{l}2.25 \mathrm{~A}^{\mathrm{a}} \\
\pm 0.95 \\
\end{array}$ & $\begin{array}{c}1.50 \mathrm{~B}^{\mathrm{ab}} \\
\pm 1.29 \\
\end{array}$ & 41.33 & $\begin{array}{c}0.75 \mathrm{~B}^{\mathrm{bc}} \\
\pm 0.96\end{array}$ & 76.34 & $\begin{array}{l}0.00 \mathrm{~B}^{\mathrm{C}} \\
\pm 0.00\end{array}$ & 100 & $\begin{array}{l}0.00 \mathrm{~B}^{\mathrm{C}} \\
\pm 0.00\end{array}$ & 100 & $\begin{array}{l}0.00 \mathrm{~B}^{\mathrm{C}} \\
\pm 0.00\end{array}$ & 100 & $\begin{array}{c}0.75 \mathrm{~B}^{\mathrm{bc}} \\
\pm 0.5\end{array}$ & 90.95 & 93.46 & 84.77 & $1.08^{\star \star}$ \\
\hline Selecrone & $\begin{array}{l}8.00 \mathrm{~A}^{\mathrm{a}} \\
\pm 6.16\end{array}$ & $\begin{array}{c}3.25 \mathrm{AB}^{\mathrm{b}} \\
\pm 2.22\end{array}$ & 64.25 & $\begin{array}{c}0.75 \mathrm{~B}^{\mathrm{b}} \\
\pm 0.5\end{array}$ & 93.35 & $\begin{array}{l}0.00 \mathrm{~B}^{\mathrm{b}} \\
\pm 0.00\end{array}$ & 100 & $\begin{array}{l}0.00 \mathrm{~B}^{\mathrm{b}} \\
\pm 0.00\end{array}$ & 100 & $\begin{array}{c}0.00 \mathrm{~B}^{\mathrm{b}} \\
\pm 0.00\end{array}$ & 100 & $\begin{array}{c}0.00 \mathrm{~B}^{\mathrm{b}} \\
\pm 0.00\end{array}$ & 100 & 98.67 & 92.93 & $3.65^{\star \star}$ \\
\hline Radiant & $\begin{array}{c}3.75 \mathrm{~A}^{\mathrm{a}} \\
\pm 1.5\end{array}$ & $\begin{array}{l}1.5 \mathrm{~B}^{\mathrm{b}} \\
\pm 1.29\end{array}$ & 64.80 & $\begin{array}{l}1.25 \mathrm{~B}^{\mathrm{b}} \\
\pm 1.26\end{array}$ & 76.34 & $\begin{array}{c}0.75 \mathrm{~B}^{\mathrm{b}} \\
\pm 1.5\end{array}$ & 88.42 & $\begin{array}{l}0.50 \mathrm{~B}^{\mathrm{b}} \\
\pm 1.00\end{array}$ & 94.47 & $\begin{array}{l}0.00 \mathrm{~B}^{\mathrm{b}} \\
\pm 0.00\end{array}$ & 100 & $\begin{array}{c}0.75 \mathrm{~B}^{\mathrm{b}} \\
\pm 0.5\end{array}$ & 94.56 & 90.76 & 86.43 & $1.66^{\star \star}$ \\
\hline Control & $\begin{array}{l}5.50 \mathrm{~A}^{\mathrm{d}} \\
\pm 4.65\end{array}$ & $\begin{array}{l}6.25 \mathrm{~A}^{\mathrm{d}} \\
\pm 4.71\end{array}$ & - & $\begin{array}{l}7.75 A^{\text {cd }} \\
\pm 5.12\end{array}$ & - & $\begin{array}{r}9.50 \mathrm{Ac} \\
\pm 4.65\end{array}$ & - & $\begin{array}{c}13.25 \mathrm{~A}^{\mathrm{bc}} \\
\pm 3.59\end{array}$ & - & $\begin{array}{c}16.25 \mathrm{~A}^{\mathrm{ab}} \\
\pm 2.22\end{array}$ & - & $\begin{array}{c}20.25 \mathrm{~A}^{\circ} \\
\pm 4.27\end{array}$ & - & - & - & $6.28^{\star \star}$ \\
\hline \begin{tabular}{|l|} 
L.S.D \\
0.05
\end{tabular} & 6.11 N.S & $4.25 \mathrm{~N} . \mathrm{S}$ & - & $4.15^{\star *}$ & - & $3.77^{* *}$ & - & $2.87^{* *}$ & - & $1.71^{* *}$ & - & $3.34^{* *}$ & - & - & - & - \\
\hline
\end{tabular}

${ }^{\star}$ Means followed the same capital letter in a column for different pesticides or small letter in row of each pesticides at different times are not significantly different at $5 \%$ level of Probability (Duncan's Multiple Rang Test).

Table (4): Efficiency of tested pesticides against larvae of Cassida vittata on sugar beet leaves during 2009/2010 at Sharkia Governorate.

\begin{tabular}{|c|c|c|c|c|c|c|c|c|c|c|c|c|c|c|c|c|}
\hline \multirow{4}{*}{ Treatment } & \multicolumn{13}{|c|}{ \%Reduction at time intervals } & \multirow{4}{*}{$\begin{array}{c}\text { Mean of } \\
\text { residual } \\
\text { effect }\end{array}$} & \multirow{4}{*}{$\begin{array}{c}\text { General } \\
\text { mean of } \\
\% \\
\text { reduction }\end{array}$} & \multirow{4}{*}{$\begin{array}{l}\text { L.S.D } \\
0.05\end{array}$} \\
\hline & \multirow{3}{*}{\begin{tabular}{|c|}
$\begin{array}{c}\text { One day } \\
\text { before } \\
\text { application }\end{array}$ \\
Mean \\
\end{tabular}} & \multirow{2}{*}{\multicolumn{2}{|c|}{\begin{tabular}{|c|} 
Initial effect \\
(after one day)
\end{tabular}}} & \multicolumn{10}{|c|}{ In days (residual effect) } & & & \\
\hline & & & & \multicolumn{2}{|c|}{3} & \multicolumn{2}{|c|}{5} & \multicolumn{2}{|c|}{7} & \multicolumn{2}{|c|}{9} & \multicolumn{2}{|c|}{11} & & & \\
\hline & & Mean & $\begin{array}{c}\text { Reduc- } \\
\text { tion }\end{array}$ & Mean & $\begin{array}{l}\text { Reduc- } \\
\text { tion }\end{array}$ & Mean & $\begin{array}{c}\text { Reduc- } \\
\text { tion }\end{array}$ & Mean & $\begin{array}{c}\text { Reduc- } \\
\text { tion }\end{array}$ & Mean & $\begin{array}{c}\text { Reduc- } \\
\text { tion }\end{array}$ & Mean & $\begin{array}{c}\text { Reduc- } \\
\text { tion }\end{array}$ & & & \\
\hline Marshal & $\begin{array}{c}49.75 \mathrm{~A}^{\mathrm{a}} \\
\pm 2.63 \\
\end{array}$ & $\begin{array}{c}11.25 \mathrm{~B}^{\mathrm{b}} \\
\pm 2.87 \\
\end{array}$ & 77.38 & $\begin{array}{l}0.00 \mathrm{~B}^{\mathrm{c}} \\
\pm 0.00 \\
\end{array}$ & 100 & $\begin{array}{l}0.00 \mathrm{~B}^{\mathrm{c}} \\
\pm 0.00 \\
\end{array}$ & 100 & $\begin{array}{l}0.00 \mathrm{~B}^{\mathrm{c}} \\
\pm 0.00\end{array}$ & 100 & $\begin{array}{l}0.00 \mathrm{~B}^{\mathrm{c}} \\
\pm 0.00 \\
\end{array}$ & 100 & $\begin{array}{l}0.00 \mathrm{~B}^{\mathrm{c}} \\
\pm 0.00 \\
\end{array}$ & 100 & 100 & 96.23 & $2.16^{\star \star}$ \\
\hline Selecrone & $\begin{array}{c}42.75 \mathrm{~A}^{\mathrm{a}} \\
\pm 3.77\end{array}$ & $\begin{array}{l}1.00 \mathrm{C}^{\mathrm{b}} \\
\pm 1.15\end{array}$ & 97.66 & $\begin{array}{l}0.00 \mathrm{~B}^{\mathrm{b}} \\
\pm 0.00\end{array}$ & 100 & $\begin{array}{l}0.00 \mathrm{~B}^{\mathrm{b}} \\
\pm 0.00\end{array}$ & 100 & $\begin{array}{l}0.00 \mathrm{~B}^{\mathrm{b}} \\
\pm 0.00\end{array}$ & 100 & $\begin{array}{l}0.00 \mathrm{~B}^{\mathrm{b}} \\
\pm 0.00\end{array}$ & 100 & $\begin{array}{l}0.00 \mathrm{~B}^{\mathrm{b}} \\
\pm 0.00\end{array}$ & 100 & 100 & 99.61 & $2.19^{\star \star}$ \\
\hline Radiant & $\begin{array}{l}49.5 \mathrm{~A}^{\mathrm{a}} \\
\pm 9.25\end{array}$ & $\begin{array}{c}12.25 \mathrm{~B}^{\mathrm{b}} \\
\pm 4.34\end{array}$ & 75.25 & $\begin{array}{l}1.25 \mathrm{~B}^{\mathrm{c}} \\
\pm 0.95\end{array}$ & 97.56 & $\begin{array}{l}0.00 \mathrm{~B}^{\mathrm{c}} \\
\pm 0.00\end{array}$ & 100 & $\begin{array}{l}0.00 \mathrm{~B}^{\mathrm{c}} \\
\pm 0.00\end{array}$ & 100 & $\begin{array}{l}0.00 \mathrm{~B}^{\mathrm{c}} \\
\pm 0.00\end{array}$ & 100 & $\begin{array}{l}0.00 \mathrm{~B}^{\mathrm{C}} \\
\pm 0.00\end{array}$ & 100 & 99.51 & 95.47 & $5.71^{\star \star}$ \\
\hline Control & $\begin{array}{c}41.75 \mathrm{~A}^{\mathrm{a}} \\
\pm 4.35 \\
\end{array}$ & $\begin{array}{c}41.75 \mathrm{~A}^{\mathrm{a}} \\
\pm 4.35 \\
\end{array}$ & 一 & $\begin{array}{c}43.25 \mathrm{~A}^{\mathrm{a}} \\
\pm 4.57\end{array}$ & 一 & $\begin{array}{c}40.25 \mathrm{~A}^{\mathrm{a}} \\
\pm 3.09\end{array}$ & 一 & $\begin{array}{c}38.00 \mathrm{~A}^{\text {ab }} \\
\pm 3.74\end{array}$ & 一 & $\begin{array}{c}34.25 \mathrm{~A}^{\mathrm{bC}} \\
\pm 2.87 \\
\end{array}$ & 一 & $\begin{array}{c}30.25 \mathrm{~A}^{\circ} \\
\pm 3.59 \\
\end{array}$ & 一 & - & - & $5.65^{\star *}$ \\
\hline \begin{tabular}{|l|} 
L.S.D \\
0.05
\end{tabular} & 8.64 N.S & $4.49^{* *}$ & - & $3.59^{* *}$ & - & $2.38^{* *}$ & - & $2.88^{* *}$ & - & $2.21^{* *}$ & - & $2.76^{* *}$ & - & - & - & - \\
\hline
\end{tabular}

*Means followed the same capital letter in a column for different pesticides or small letter in row of each pesticides at different times are not significantly different at $5 \%$ level of Probability (Duncan's Multiple Rang Test). 
J. Plant Prot. and Pathology, Mansoura Univ., Vol. 2 (6), June, 2011 